Ann. Biol. anim. Bioch. Biophys., I969, 9 (2), 219-232.

\title{
LIEU DE LA FORMATION DES CHYLOMICRONS ; LEUR IMPORTANCE POUR L'ABSORPTION NORMALE DES ACIDES GRAS A GHAINE LONGUE. ASPEGT MORPHOLOGIQUE
}

\author{
N. VODOVAR, J. FL.ANZY et A.-C. FRANÇOIS \\ Station centrale de Nutrition, \\ Centre national de Recherches zootechniques, 78 - Jouy-en-Josas \\ Institut national de la Recherche agronomique
}

\section{SOMMAIRE}

Le lieu de la formation des chylomicrons dans la cellule intestinale absorbante au cours de l'absorption des acides gras à châ̂ne longue a été étudié chez le Porc. Le mécanisme de la formation de ces particules, leur composition, leur taille et leur acheminement à travers la cellule ont été interprétés et discutés.

Des perturbations morphologiques dans le cas de non-formation de chylomicrons ont été observées après administration au Rat et au Porcelet de cycloheximide, qui, en inhibant la synthèse protéique, empêche, dans les conditions d'expériences, la formation de chylomicrons.

\section{INTRODUCTION}

Les particules, morphologiquement semblables, présentes dans la lymphe et dans le sang penđant l'absorption des lipides et à des temps variables après l'absorption, furent appelées par GuILIVER (I840) " molecular base of the chyle ". En étudiant la nature et la taille de ces particules dans le sang, puis dans la lymphe, GaGE (I920), observa, en microscopie optique, que l'apparition de ces particules se fait d'abord dans le chyle, situa leur diamètre entre 0,5 et I $\mu$ et leur donna le nom de chylomicrons. Cette appellation, peu satisfaisante, doit être néanmoins conservée pour ne pas ajouter davantage de confusion dans la nomenclature des lipides. D'autres études et, en particulier durant les quinze dernières années, ont été faites chez des espèces différentes par des méthodes biochimiques, biophysiques et histologiques (DOLE, HAMLIN, I962) pour déterminer l'aspect, la taille, la structure et la composi- 
tion des chylomicrons isolés de la lymphe et du sang, prélevés après ingestion de lipides. On s'accorde pour considérer que la forme des chylomicrons est sphérique, qu'une enveloppe plus opaque est présente régulièrement à la périphérie, que la taille des chylomicrons prélevés au cours d'un repas gras est variable quelle que soit la nature des graisses ingérées (GAGE, FISH, I924; CASLEY-SMITH, I962; PINTER, Zilversmit, I962 ; YokOYAMA, ZILVERSMIT, I965; QUARFORDT, GoOdMAN, I966 ; Vodovar, Flanzy, Françors, 1967) et que la valeur du diamètre de la plupart de ces particules est généralement comprise entre 0,1 et $0,5 \mu$ avec des valeurs extrêmes - sauf exception - de 0,06 et I $\mu$. En ce qui concerne la taille des chylomicrons provenant de graisses de différentes compositions, ingérées séparément, les avis sont souvent partagés et souvent contradictoires (Thomas, ScoTT, I962 ; CoUREL, Clé́MeNT, I964; Zilversmit, Sisco, Yokoyama, I966). En fait, il semble que le diamètre moyen d'un ensemble de chylomicrons, présents dans la cellule absorbante ou dans la lymphe durant l'absorption, peut varier suivant la nature des repas gras, mais il semblerait que dans la plupart des cas ces variations demeurent peu importantes (Vodovar, Flanzy, FrançoIs, I967). Malgré les difficultés d'isolement des chylomicrons proprement dits, les résultats de leur analyse chimique ont montré que les triglycérides représentent, grossièrement, 90 à $95 \mathrm{p}$. I00 de substance et qu'ils sont associés à des phospholipides, du cholestérol libre ou estérifié et à des protéines (LAUReli, I953; LaUreli, I954; Savary, Constantin, Desnuelite, i96I ; MatTson, VolPENHEIN, I964; Zil,VERSMiT, I967). La présence de ce dernier constituant, actuellement bien établie (BoRGSTröm, I96o) fut, pendant un certain temps, considérée comme substance non intégrante des chylomicrons du fait de son élimination par des lavages alcalins répétés des chylomicrons.

L'architecture suivant laquelle les différents éléments des chylomicrons sont répartis, n'a pas encore été définitivement établie ; cependant, on s'accorde généralement à penser que la partie centrale est formée par des triglycérides avec lesquels, suivant l'avis de certains, sont mélangés les phospholipides et le cholestérol (LINDGREN, NICHOLS, I960), tandis que, pour d'autres chercheurs, ces dernières substances seraient à la périphérie des triglycérides (DOLE, HAML,IN, I962).

Pour les uns et les autres, les protéines forment l'enveloppe la plus externe, mais, suivant certains, les phospholipides, le cholestérol non estérifié et même une faible fraction des triglycérides sont associés aux protéines pour l'édification d'une enveloppe mosaïque (ZiLversmit, I965), tandis que les triglycérides et le cholestérol estérifié seraient à l'intérieur des chylomicrons.

De nombreux chercheurs sont d'avis que les acides gras des triglycérides des chylomicrons sont, en majeure partie, d'origine alimentaire (BoRGSTRöM, I95I ; Bergström, Borgström, Carlsten, I954 ; Bi,omstrand, Ahrens Jr, I958 ; Bragdon, Karmen, i96o ; Savary, Constantin, Desnuel, RY, I965). Toutefois, le pourcentage et l'origine des acides gras non alimentaires sont toujours controversés (Karmen, Whyte, Goodman, I963 ; Savary, Constantin, I966). L'absence de phospholipides et de cholestérol dans le repas gras ne semble pas influencer la formation des chylomicrons, ce qui indiquerait que 1'origine de ces éléments peut être exclusivement endogène, mais leur réelle provenance n'est pas encore élucidée. Les protéines des chylomicrons (BRAGDON, I958 ; RODBELI, FrEDERICKSON, ONO, I959 ; RODBELL, FREDERICKSON, I959) semblent pouvoir être incluses dans les lipoprotéines (SCANU, PAGE, I959; DoLE, HAMLIN, I962) sans toutefois que leur 
nature soit, à 1'heure actuelle, déterminée d'une façon précise. En revanche, il est bien établi (Isselbacher, Budz, I963; HATCh, Hagopian, RUBENSTEIN, I966 ; ROHEIM, GIDEz, EDER, I966) que la muqueuse intestinale et plus précisément les cellules absorbantes, peuvent synthétiser les protéines de la même nature que celles qui forment la partie constituante des chylomicrons. Les nombreuses observations indiquent que l'absence congénitale de $\beta$-lipoprotéines d'une part (REY, I96I ; FREZAL, et al., I96I ; ISSELBACHER et al., I964 ; LÉVY, FREDRICKSON, LASTER, I966 ; DobBins, I966 ; Ways et $a l .$, I967) et l'inhibition de la synthèse protéique au cours de l'absorption des lipides, d'autre part (SABESIN, ISSELBACHER, I965; VodOvaR, MAssicard, Flanzy, Ig68) provoquent des troubles de l'absorption des lipides qui se traduisent par l'absence de chylomicrons dans la lymphe.

La plupart des travaux concernant les chylomicrons sont du domaine de la biochimie et ont trait à la composition et à la taille de ces particules prélevées soit dans la lymphe soit dans le sang. Cependant, il est généralement admis que l'apparition de ces particules se fait déjà dans la cellule absorbante (VoDovar, FLANzY, I967) mais les renseignements sur le lieu et le mécanisme de leur formation, ainsi que sur le rôle qu'elles jouent pour l'acheminement des lipides à travers la cellule absorbante, ne sont pas connus.

Par les présentes observations, nous avons essayé de localiser le niveau exact de l'apparition des chylomicrons dans la cellule absorbante au cours de l'absorption normale des acides gras à chaîne longue ; les chaînes grasses courtes forment peu de chylomicrons et transitent généralement à travers le cytoplasme de la cellule absorbante sous forme libre (Vodovar, FLANzY, I967). Ensuite, nous avons étudié les conséquences de l'inhibition de la synthèse protéique par la cycloheximide sur la formation des chylomicrons et sur l'acheminement des chaînes grasses à travers la cellule absorbante et leur évacuation vers le stroma. Fnfin, nous avons observé l'aspect de l'accumulation provoquée des chylomicrons par pincement des villosités intestinales pendant l'absorption normale des acides gras et fait la comparaison avec l'accumulation des glycérides en absence de formation de chylomicrons. Compte tenu des résultats obtenus, la relation probable entre le lieu de formation des chylomicrons et leur composition a été discutée. Les causes possibles de non formation des chylomicrons et les conséquences ont été discutées et interprétées.

\section{MATÉRIEL ET MÉTHODES}

Les fragments de l'intestin grêle ont été prélevés sur les porcelets et les porcs de race Large White, d'âge variable, et sur les rats de souche Wistar, pesant environ $15^{\circ} \mathrm{g}$; cette dernière espèce nous a servi surtout pour la mise au point des techniques.

Pour les observations sur le lieu de formation des chylomicrons, les animaux mis au régime lipidoprive pendant 3 jours, puis privés de nourriture pendant 16 à 24 heures, ont reçu un des acides expérimentés $\left(\mathrm{C}_{16}, \mathrm{C}_{18}, \mathrm{C}_{18: 1}, \mathrm{C}_{18: 2}\right)$ soit par ingestion, soit par infusion dans un segment intestinal isolé (VODOVAR, FLANZY, 1966).

Chaque prélèvement a été effectué sur un animal anesthésié par injection intrapéritonéale de nembutal ou mis sous narcose par l'éther ou le chloroforme. Quatre abattages d'animaux ont été pratiqués, à intervalle de 20 minutes après l'ingestion et de to minutes après l'infusion dans le segment isolé.

Pour les observations sur l'action de la cycloheximide, les animaux ayant reçu le régime indiqué précédemment, la cycloheximide a été administrée par injection intrapéritonéale à raison de $10 \mathrm{mg}$ 
(dissous dans $2 \mathrm{ml}$ de $\mathrm{ClNa}$ à 0,9 p. Ioo) par ıoo g de poids vif. Les témoins ont reçu $2 \mathrm{ml}$ de ClNa a $0,9 \mathrm{p}$. roo. Trente minutes, 2 heures, puis 4 heures après administration de cycloheximide, les différents lots d'animaux ont reçu soit un acide gras expérimenté $\left(\mathrm{C}_{4}, \mathrm{C}_{8}, \mathrm{C}_{12}, \mathrm{C}_{16}, \mathrm{C}_{18: 1}, \mathrm{C}_{18: 2}\right)$, soit un mélange de plusieurs acides. Les témoins ont reçu une quantité équivalente dans les mêmes conditions. Les prélèvements ont été faits dans les intervalles de temps déjà indiqués.

L'obstruction à l'évacuation des chylomicrons par pincement à la base des villosités a été faite sur des animaux sous anesthésie après infusion de l'acide gras dans le segment isolé.

La fixation par faible extension in situ a été faite soit au tétroxyde d'osmium à 2 p. 100, tamponné phosphate (Millonig, 196I) pendant 5 minutes, puis pendant I à 2 heures à $4^{\circ} \mathrm{C}$, soit au glutaraldéhyde à $6 \mathrm{p}$. roo avec postfixation dans le tétroxyde d'osmium comme précédemment.

Les tissus ont été déshydratés dans l'acétone et !'inclusion a été faite dans l'Epon 812 (LuFT, I96I). Les blocs ont été coupés avec l'Ultratome III LKB, à une épaisseur comprise entre $45^{\circ}$ et $550 \AA$.

Les coupes, contrastées sur grille avec l'acétate d'uranyle (Reynolds, 1963) 15 minutes, et avec le citrate de plomb, Io minutes, ont été observées au Siemens Elmiscop I.

\section{OBSERVATIONS}

Nous avons déjà étudié le mécanisme de l'absorption des graisses chez le Porc (Vodovar, Flanzy, I966). A cette occasion, nous avons rappelé et discuté les différents travaux concernant l'aspect morphologique de l'absorption des lipides. Nous ne reviendrons pas sur ces résultats, les présentes observations étant limitées aux chylomicrons.

\section{Lieu de formation des chylomicrons}

La localisation du lieu de formation des chylomicrons présente des difficultés du fait que les acides gras à chaîne longue sont acheminés à travers la cellule absorbante à l'intérieur du RER, sauf pendant leur transit dans les vésicules golgiennes. La paroi réticulaire autour des particules lipidiques, qui sont en général isolées, est toujours distendue et intimement associée à la presque totalité de la surface de ces particules.

Aspect de la section des particules lipidiques dont les coupes sont perpendiculaires ou obliques sur l'axe du RER.

Sur une telle section de la particule lipidique, dès sa pénétration dans l'endoplasme, on observe une surface circulaire, relativement homogène, entourée d'une couche opaque plus ou moins épaisse, rarement uniforme (fig. I). La différence d'aspect d'une même couche et entre les couches de différentes particules s'explique par 1'incidence des coupes sur l'axe du RER dont la paroi est inégalement distendue autour des particules. Sur les coupes perpendiculaires à l'axe du RER et passant par le centre des gouttelettes, les couches opaques sont relativement uniformes, leur épaisseur est la plus faible (fig. I). Cela est dû au fait que ces coupes sectionnent la paroi réticulaire verticalement à leur axe et aux points où elle est le plus distendue. Sur les coupes également perpendiculaires à l'axe du RER, mais ne passant pas par le centre des particules, les couches opaques demeureront assez uniformes mais leur épaisseur sera supérieure à celle des coupes précédentes, la paroi réticulaire étant moins distendue. Pour toutes les coupes dont l'incidence est oblique sur l'axe du RER, l'épaisseur des couches opaques est inégale et non homogène. Cet aspect général des coupes des particules lipidiques est semblable à tous les niveaux de l'endo- 
plasme, excepté pour celles présentes dans les vésicules golgiennes (fig. 2). Les gouttelettes lipidiques, toujours abondantes dans ces vésicules au cours de l'absorption normale des lipides, sont pourvues d'une enveloppe bien distincte de la couche opaque observée, quand ces particules se trouvent entourées du RER ; cette enveloppe n'est pas une cyto-membrane mais un pourtour formé de substance plus osmiophile que l'intérieur des particules. Elle permet à ces dernières de demeurer en contact, tout en conservant leur individualité. Les particules observées dans les vésicules golgiennes sont identiques à celles présentes, plus loin, dans les espaces intercellulaires (VoDoVAR, Flanzy, I966, fig. II) et dans le stroma des villosités (Vodovar, FLANzY, I967, fig. 7 et Io) et présentent tous les caractères morphologiques des chylomicrons.

Section des particules lipidiques dont les coupes sont parallèles à l'axe du RER.

$\mathrm{Au}$ cas où l'incidence de la coupe d'une particule lipidique est parallèle à l'axe de la lumière réticulaire, dans laquelle est située la particule, et à condition que cette coupe traverse la lumière réticulaire au moins d'un côté de la particule (fig. 3), on observe qu'une partie de la surface de la particule limite la lumière réticulaire et, de ce fait, n'est pas en contact avec le RER. Sur cette partie de la périphérie non associée à la paroi réticulaire, on observe la présence de la bordure opaque. Cette bordure est variable suivant 1 'incidence des coupes et toujours de même aspect que l'enveloppe se trouvant autour des chylomicrons.

Ces observations, à condition que l'incidence des coupes soit semblable, sont identiques aussi bien sur la section des gouttelettes dans l'endoplasme, immédiatement sous la zone apicale, que pour les particules ayant déjà transité à travers les vésicules golgiennes.

Sur la nature de cette enveloppe et sur son rôle dans le transit des triglycérides à travers la cellule absorbante, nous reviendrons plus loin. Cependant, on peut affirmer dès maintenant que cette bordure, qui permet d'identifier les chylomicrons, se forme au cours du passage des triglycérides de la zone apicale dans l'endoplasme. Ces constatations sont également confirmées par les coupes sur lesquelles, aussi bien dans la zone apicale que plus loin dans l'endoplasme, on trouve deux ou plusieurs gouttelettes, côte à côte, à l'intérieur du RER (fig. 4), mais qui, grâce à la présence de leur enveloppe, ne s'agglomèrent pas. De même, parfois, on observe des chylomicrons dans les espaces intercellulaires immédiatement sous la zone apicale (fig. 5). Par ailleurs, on trouve, quoique assez rarement, des formes osmiophiles étalées soit à l'intérieur, soit à l'extérieur du réticulum, mais leur aspect est nettement distinct de celui des chylomicrons.

\section{Taille et acheminement des chylomicrons}

Entre la forme imprécise de la substance osmiophile dans la zone apicale et l'apparition des chylomicrons dans l'endoplasme, on n'a pas observé d'étapes intermédiaires. La simultanéité et la rapidité de l'édification de ces particules masquées par le RER en sont des raisons possibles. Au fur et à mesure que les chylomicrons apparaissent dans l'endoplasme, on observe, aussi bien sur les coupes parallèles au grand axe de la cellule que sur les coupes perpendiculaires, que leur acheminement se fait, sauf exception, isolément et d'une façon bien régulière (fig. I à 5). Si l'on tient compte du nombre des chylomicrons à différents niveaux des cellules et dans les 
espaces intercellulaires d'une villosité, et de l'aspect du réticulum dans ces cellules, on peut présumer que le rythme de formation et d'acheminement des chylomicrons est plus rapide pour les cellules occupant la partie distale des villosités. Il décroît progressivement en se rapprochant de la base des villosités. Les chylomicrons observés au cours de l'absorption d'un acide gras donné dans la cellule absorbante, à un moment donné de l'absorption, semblent être de taille relativement homogène. Si l'on tient compte de l'incidence des coupes, cette homogénéité semble se retrouver également chez une population de cellules absorbantes occupant des positions homologues sur les villosités. En revanche, il semble que la taille des chylomicrons soit variable suivant le niveau auquel les cellules sont prélevées sur les villosités. Contrairement à ce que l'on observe en ce qui concerne la rapidité de formation et d'acheminement, la taille des chylomicrons paraît devenir plus grande au fur et à mesure qu'on observe des cellules absorbantes se rapprochant de la base des villosités. L'interprétation de ces observations devient encore plus complexe si on compare l'aspect à différents moments après l'ingestion des acides gras.

\section{Action de la cycloheximide sur le mécanisme de l'absorption des acides gras}

Les coupes de tissus de la paroi intestinale prélevés dans les conditions habituelles sur des animaux ayant ingéré des acides gras à chaîne longue, une demi-heure après que leur a été administrée, par injection intrapéritonéale, la cycloheximide, ont été observées. L'aspect des cellules absorbantes intestinales, compte tenu des temps de prélèvement après l'ingestion des acides, se trouve plus ou moins fortement modifié par l'accumulation de glycérides dans l'endoplasme (fig. 6).

La structure des microvillosités et des zones apicales paraît inchangée au moins au début de l'absorption. La pénétration des acides gras à travers la membrane plasmique libre, leur estérification et leur acheminement à travers la zone apicale semblent s'effectuer apparemment de la même façon que chez les animaux témoins, qui n'ont pas reçu de cycloheximide (fig. 6). La rapidité de 1'apparition des glycérides dans l'endoplasme, et toujours à l'intérieur du RER, ne semble pas être modifiée, tandis que leur forme et leur acheminement à travers la cellule absorbante sont, au fur et à mesure de l'absorption, de plus en plus perturbés. Au début de l'absorption, les premières gouttelettes de glycérides parvenues dans l'endoplasme sont isolées, de forme assez irrégulière et de taille très variable, pour la plupart supérieure à I $\mu$. La couche opaque externe, qui les entoure, est nettement différente des coupes témoins sans cycloheximide. Ces gouttelettes augmentent de volume au fur et à mesure de 1'arrivée dans l'endoplasme des glycérides, qui ne s'évacuent pas. La paroi des différents tubes réticulaires se distend de plus en plus par accumulation de glycérides ; les parois parviennent au contact les unes des autres par leur surface extérieure, donnant parfois l'impression que les glycérides se trouvent dans les espaces entre les tubes réticulaires et non à 1'intérieur (fig. 6, 7, 8, 9). Par la suite, les parois réticulaires se rompent les unes après les autres, tandis que les glycérides s'agglomèrent en particules de plus en plus volumineuses (fig. $7,8,9$ ), de forme plus ou moins régulière, de diamètre souvent supérieur à Io $\mu$. Ainsi, le diamètre de ces particules tend à se confondre avec celui de la cellule. Les éléments cytoplasmiques, à ce stade, sont comprimés entre la masse des glycérides ou repoussés vers la périphérie de la 
cellule (fig. 9). Les noyaux, pour la plupart, ont un périmètre multilobé (fig. 8), les mitochondries paraissent modifiées ou en état de disparaître. Le RER est repoussé sur la périphérie des particules lipidiques (fig. 7, 9), les ribosomes paraissent souvent de taille inférieure à celle des sujets normaux, qui n'ont pas reçu de cycloheximide ; ils paraissent souvent détachés de la paroi réticulaire et se trouvent tantôt disséminés, tantôt groupés en amas (fig. 6, 7, 9). Les vésicules golgiennes, qui, au cours de l'absorption normale, sont engorgées de lipides, demeurent vides (fig. 7), aplaties quelle que soit l'incidence des coupes. L'envahissement de la cellule absorbante par les glycérides est d'abord observé dans la partie supranucléaire, puis rapidement dans la région périnucléaire et sous les noyaux, de façon telle que la cellule absorbante prend l'aspect d'une cellule adipeuse. Au stade de l'accumulation des lipides, des éléments semblables aux lysosomes commencent à apparaître aux différents niveaux de 1'endoplasme (fig. 6). Les espaces entre les cellules absorbantes sont peu importants et sans chylomicrons (fig. 7, 9). On constate également l'absence de chylomicrons dans le stroma des villosités. La basale, qui sépare la couche épithéliale du stroma, paraît subir des modifications importantes.

Ces observations montrent que le défaut de synthèse protéique provoque des troubles graves de l'absorption des lipides par accumulation de triglycérides en l'absence de formation de chylomicrons. On peut dire que les phospholipides et le cholestérol ne suffisent pas à maintenir les gouttelettes isolées et à assurer leur transport.

Sur les coupes de tissus prélevés sur les animaux ayant ingéré des acides gras à chaîne courte $\left(\mathrm{C}_{4}, \mathrm{C}_{6}, \mathrm{C}_{8}\right)$, l'aspect morphologique est semblable à celui des témoins n'ayant pas reçu de cycloheximide. Cela indique que les chaînes grasses libres - ce qui est le cas pour les chaînes courtes car elles ne s'estérifient pas, en général peuvent transiter à travers le cytoplasme et que l'inhibition de la synthèse protéique n'a pas d'action sur leur absorption.

\section{Accumulation provoquée des chylomicrons dans la cellule absorbante et dans le stroma des villosités}

$\mathrm{Si}$, au cours de l'absorption des acides gras à chaîne longue dans un segment isolé, sous anesthésie, on parvient à empêcher l'évacuation des glycérides d'une ou plusieurs villosités par pincement à leur base avec des pinces fines, on provoque leur accumulation (fig. Io, II). Cette accumulation est observée à tous les niveaux, aussi bien immédiatement sous la zone apicale que dans les vésicules golgiennes, les espaces intercellulaires et le stroma des villosités. L'ensemble de ces accumulations, quel que soit le lieu, est, dans un premier temps, caractérisé par le rassemblement des particules entourées d'une enveloppe, qui caractérise les chylomicrons et qui les protège contre l'agglomération (fig. 4, 5, Io, II). En supprimant la cause de l'empêchement de l'évacuation, c'est-à-dire en enlevant les pinces, et en faisant le prélèvement une dizaine de minutes plus tard, on constate que l'évacuation des chylomicrons est reprise et qu'un équilibre tend à se rétablir à 1'intérieur de la cellule absorbante. Si toutefois l'empêchement a été prolongé pendant plus d'une heure, la structure des tissus des villosités donne le signe d'une dégradation. De nombreux globules rouges apparaissent hors des capillaires, les chylomicrons commencent à perdre leur individualité par dégradation de l'enveloppe qui les entoure et on trouve, 
au même endroit, l'accumulation des chylomicrons et l'agglomération des glycérides qui ne sont plus sous forme de chylomicrons. A un stade ultérieur, la plupart des particules ont perdu leur aspect de chylomicrons.

Ces observations confirment que les chylomicrons sont édifiés dès le passage des glycérides dans l'endoplasme et, que la couche externe permet à ces particules de garder leur individualité (fig. Io, II), ce qui les distingue nettement des glycérides accumulés dans le cas de non formation de chylomicrons (fig. $7,8,9$ ).

\section{DISCUSSION}

\section{Lieu de formation des chylomicrons et origine probable de leurs acides gras}

Les présentes observations montrent que la formation des chylomicrons, pendant l'absorption normale des acides gras à chaîne longue, est située soit au passage des triglycérides de la zone apicale dans l'endoplasme, soit dès leur apparition dans l'endoplasme, ce qui, pour l'instant, revient au même, car la limite entre les deux zones reste imprécise. L'inhibition de la synthèse protéique par la cycloheximide empêche la formation de chylomicrons dans la cellule absorbante en provoquant 1'accumulation des triglycérides. Cette accumulation commençant à apparaître immédiatement sous la zone apicale, confirme, si cela était nécessaire, le lieu de formation des chylomicrons. Cette localisation du lieu de la formation des chylomicrons implique que l'estérification ou la ré-estérification des acides gras qui entrent dans la composition des chylomicrons est située obligatoirement dans la zone apicale. Ceci est en accord avec certains travaux sur ce sujet (Forstner et al., I965; Vodovar, Flanzy, I966 ; Sjöstrand, BoRgström, I967).

Les acides gras des triglycérides incorporés dans les chylomicrons formés au niveau indiqué proviendraient surtout de la lumière intestinale. Il est, en effet, peu probable qu'une quantité importante des acides gras de la circulation sanguine ou lymphatique vienne en contresens de l'absorption dans la zone apicale de la cellule absorbante pour être estérifiée et faire partie des chylomicrons. De même, il semble que la synthèse des acides gras, sans doute possible dans la cellule absorbante (FRANKS, RILEY, ISSELBACHER, I966), ne soit pas assez active à ce niveau au cours de l'absorption pour influencer sensiblement la composition des chylomicrons en acides gras.

Les chaînes grasses de provenance intraluminaire sont soit d'origine exogène, c'est-à-dire qu'elles proviennent des aliments ingérés, soit d'origine endogène, c'està-dire qu'elles ont pour source la bile, le suc pancréatique, l'excrétion de la paroi intestinale et les cellules desquamées. Malgré l'importance que certains travaux attribuent aux lipides de ces différentes provenances endogènes (PESSOA, KIM, IVY, I953; WELD, I96I ; BURR, MCPhERSON, TIDWELL, I960), et, en particulier aux lipides hépatiques (Shrivastava, Redgrave, Simmonds, I966; BaXter, I966), la part des lipides endogènes provenant de la lumière intestinale et participant à la composition des chylomicrons doit être considérée comme faible par rapport aux 
acides d'origine alimentaire. S'il en était autrement, il y aurait formation continue de chylomicrons dans la cellule absorbante à partir de ces acides et leur observation serait possible à tous moments ; or, il n'y a pas formation de chylomicrons dans la cellule absorbante en dehors des repas gras.

Cela amènerait à considérer qưe les triglycérides des chylomicrons au niveau de la cellule absorbante sont surtout formés par les acides gras alimentaires. Cela n'implique pas que le rapport quantitatif entre les acides gras ingérés et ceux des triglycérides des chylomicrons soit le même. E,n effet, certaines chaînes grasses peuvent rester non estérifiées en quantité supérieure à d'autres (Vodovar, Fi.ANzY, Françors, I967, $a$ et $b$ ) ou bien l'incorporation dans les phospholipides (BLOMSTRAND, DÄHLBACK, LINDER, I959) ou les esters du cholestérol (ClÉMENT, MEAD, I959) n'est pas la même pour les différents acides gras. De même, il est possible que certaines chaînes soient métabolisées dès leur pénétration dans la cellule.

En se référant à de nombreux travaux (BorGström, I95I ; BLOMSTrand, AhRens Jr, I958 ; Savary, Constantin, Desnuelle, I96r ; Constantin, Savary, I965), on peut constater que les acides gras des triglycérides des chylomicrons prélevés dans la lymphe thoracique sont également en majorité d'origine alimentaire. Ceci engagerait à penser qu'il n'y a pas de modification notable dans la composition des chylomicrons entre la cellule absorbante et le canal thoracique. Cependant, certains travaux montrent, en revanche, que les acides gras d'origine endogène représentent parfois 43 p. Ioo des triglycérides des chylomicrons (KARMEN, WHYTE, GoOdman, I963).

Les derniers résultats sont difficiles à expliquer du point de vue morphologique compte tenu du lieu de formation des chylomicrons et du mécanisme de leur transport jusqu'au stroma (VoDOVAR, FLANZY, I966). Dans le cas où ils seraient confirmés, il faudrait envisager une modification de la composition des chylomicrons au cours de leur acheminement dans la lymphe ou bien un isolement incomplet de ces particules.

\section{Taille des chylomicrons et vitesse apparente de leur acheminement}

La connaissance du lieu de la formation des chylomicrons permet de déduire que l'édification de ces particules est faite à partir des glycérides en transit rapide et non à partir de leur accumulation comme cela aurait pu être si cette formation avait lieu dans les vésicules golgiennes. Cela indique aussi que la taille des chylomicrons et leur rapidité de transit dans la cellule absorbante sont liées à de nombreux facteurs très complexes. Si on admet, très grossièrement, que la cellule absorbante se comporte pendant l'absorption comme une pompe (VERZAR, MCDoUGALI, I936), on peut présumer que la taille des chylomicrons dépend de la quantité de triglycérides qui parviennent dans un tube réticulaire au lieu de leur formation, dans l'intervalle de temps qui sépare deux mouvements consécutif de la cellule absorbante.

La taille des chylomicrons, pour une cellule donnée, dépendra donc à la fois de la rapidité d'arrivée des glycérides dans un réseau du réticulum et de la rapidité des mouvements de la cellule. La rapidité d'arrivée des glycérides au lieu de formation des chylomicrons doit être considérée comme étant liée à des mécanismes très complexes tels que la pénétration des acides gras à travers la membrane plasmique libre, leur estérification ou ré-estérification et probablement l'interdépendance des deux 
mécanismes. Pour une rapidité déterminée avec laquelle les glycérides, toujours pour un tube réticulaire, parviennent au lieu de formation des chylomicrons, la taille de ces particules sera d'autant plus petite que les mouvements de la cellule sont plus rapides. Pour l'interprétation de ces différentes observations, il paraît nécessaire, en premier lieu, de tenir compte de l'état fonctionnel de la cellule absorbante c'est-à-dire de sa maturation, qui, comme il l'a été montré (VoDovar, FléchoN, I966 ; MERRILL, SpRINz, Tousimis, I967) se traduit par l'âge des cellules absorbantes, donc par leur position sur la villosité. Aussi, les cellules absorbantes occupant la partie distale des villosités ont-elles leur ultrastructure plus hautement évoluée, en relation avec leur fonction, que les cellules plus jeunes à la base des villosités, ce qui permet de comprendre que le fonctionnement de certaines cellules soit plus rapide et plus perfectionné. De même, la richesse enzymatique variable suivant la position que les cellules occupent sur les villosités (NoRDSTrom, DAHLQvist, JosEFson, I967) nous permet. de concevoir que les réactions biochimiques, et notamment l'estérification, puissent. être plus ou moins importantes suivant la maturation cellulaire. Les implications de différences structurales et enzymatiques sur le fonctionnement de la cellule absorbante aux différents niveaux de la villosité nous permettent de mieux comprendre la variation de la taille des chylomicrons au cours de l'absorption d'un même acide gras, d'une part, et la vitesse de leur acheminement, d'autre part. On peut également envisager que les acides gras, suivant leurs propriétés, puissent franchir les différentes étapes de 1'absorption telles que la pénétration à travers la membrane plasmique libre ou l'estérification à des temps plus ou moins longs. Ceci se traduira par un transit plus ou moins rapide à travers la cellule absorbante au même degré de maturation et une taille des chylomicrons plus ou moins grande.

\section{Non formation des chylomicrons pendant l'absorption des acides gras}

L,es différentes étapes de 1'absorption des acides gras à chaîne longue sont la pénétration à travers la membrane plasmique, l'estérification et le passage dans le réticulum, la formation des chylomicrons et leur évacuation vers le stroma. Si le fonctionnement de l'une ou de plusieurs de ces étapes est entravé ou défectueux, il $\mathrm{y}$ a perturbation dans l'absorption ou malabsorption (ISSELBACHER, I967).

L'inhibition de la synthèse protéique par différentes substances et notamment. par la cycloheximide ('Trakatelis, MonTJar, Axelrod, I965 ; Sabesin, IsselBACHER, I965) a déjà été étudiée. Le fait que l'administration de cycloheximide peu de temps avant 1'ingestion des acides gras se traduise par la non formation des chylomicrons montre que les protéines sont indispensables à la formation de ces particules. Cela indique également que la synthèse de ces protéines se fait pendant l'absorption même des acides gras, sans doute dans la cellule absorbante. L'accumulation des glycérides en l'absence de formation de chylomicrons provoque la distension, puis le déchirement de la paroi réticulaire. Cela désorganise la voie de transit des glycérides et coupe leur passage dans les vésicules golgiennes. Un aspect identique est observé sur les coupes de la muqueuse intestinale prélevée par biopsie au début du jéjunum après ingestion, d'huile de maîs chez des enfants suivis cliniquement pour malabsorption des lipides (observations non publiées). Précisons qu'il s'agit, d'une part, de sujets caractérisés par 1'absence congénitale de $\beta$-lipoprotéines et, d'autre part, de sujets dont les $\beta$-lipoprotéines circulantes demeurent normales. Les obser- 
vations, en ce qui concerne la malabsorption des glycérides en l'absence de $\beta$-lipoprotéines, concordent avec les travaux déjà faits sur le sujet (SABESIN, ISSELBACHER, I965; Dobbins, r966).

Etant donné que la non formation de chylomicrons provoque des perturbations dans le transit, on pourrait s'attendre à ce que la pénétration des acides gras dans la la cellule, leur estérification et leur passage dans le RER soient ralentis et perturbés. Or, il semble qu'il n'y a pas de modification dans ces étapes jusqu'à ce que la cellule se remplisse entièrement de glycérides. Ceci veut dire que ces différents mécanismes sont indépendants. En revanche, toutes les étapes qui suivent celle de la non formation des chylomicrons sont perturbées et modifiées. Nous ne connaissons pas actuellement le mécanisme qui permet aux glycérides agglomérés dans la cellule absorbante de parvenir dans le stroma, mais compte tenu de la formation de nombreux corps denses (lysosomes?) dans la cellule absorbante et du fait que les acides gras se retrouvent dans le stroma sous forme libre et sont acheminés en grande partie par la voie portale (KAYDFN, MEDICK, I967), on peut penser qu'en l'absence de formation de chylomicrons, l'hydrolyse des glycérides se fait dans la cellule absorbante en permettant aux acides sous forme libre de franchir la membrane plasmique et la basale (Vodovar, Flanzy, I967).

L'aspect de l'accumulation des glycérides dans la cellule absorbante, s'il n'y a pas formation de chylomicrons et dans le cas de l'accumulation provoquée des chylomicrons, est nettement différent et permet de comprendre le mécanisme de transport des glycérides dans la cellule absorbante.

Le fait que le défaut de synthèse protéique, nécessaire à la formation des chylomicrons, provoque la malabsorption des chaînes grasses longues fait supposer, comme hypothèse de travail, que le manque de phospholipides ou de cholestérol, qui sont également constituants de ces particules, et dont la provenance n'a pas encore été élucidée pour l'édification des chylomicrons, provoquerait le même effet. Dans le cas contraire, cela montrerait que le rôle des protéines est privilégié pour le transport des glycérides dans la cellule absorbante.

En conclusion de ces observations, on pourrait dire que la formation des chylomicrons se fait immédiatement après l'estérification des acides gras à chaîne longue dans la zone apicale de la cellule absorbante. La non formation des chylomicrons provoque des troubles graves dans l'absorption, le défaut de protéines est la cause, en toute probabilité, la plus fréquente de l'absence de ces particules après l'ingestion d'un repas gras. Les acides gras à chaîne courte et la grande majorité des chaînes grasses moyennes ne sont pas estérifiés et comme tels transitent à travers le cytoplasme sans occasionner de troubles de digestion et il faut en tenir compte pour que, dans certains cas de malabsorption des lipides, le régime soit mieux adapté.

Reģu pour publication en novembre 1968.

\section{REMERCIEMENTS}

Nous remercions le docteur H.-L. VINCENT de la Upjohn International Company, Kalamazoo, Michigan qui nous a offert la cycloheximide (Acti-dione) et les Laboratoires Astra-calvé, Asnières, qui nous ont gracieusement fourni les acides gras. 


\title{
SUMMARY
}

\author{
THE CHYLOMICRONS OF THE INTESTINAL ABSORPTIVE CELL : \\ SITE OF FORMATION, FINE STRUCTURE \\ AND FUNCTION IN THE MECHANISM OF LONG CHAIN FATTY ACID ABSORPTION
}

The site of chylomicron formation in the intestinal absorptive cell has been studied on pigs fed free or esterified long chain fatty acids. Absence of chylomicron formation was recorded, and its effect on glyceride transport was studied on piglets and rats administered with cycloheximide as an inhibitor of protein synthesis during lipid absorption.

Long-chain fatty acids, esterified or re-esterified in the terminal web after passing through the free plasmic membrane, appeared in the endoplasm inside the rough endoplasmic reticulum (RER), in the form of droplets sheathed in an opaque membrane mostly connected, either partly or totally, with the surrounding reticular wall. In the disconnected particles, this membrane is morphologically identical with the opaque film encircling the fat particles that have reached the Golgi complex, intracellular space and stroma of the villi. This membrane is tipical of chylomicrons and allows the clustering of definitely separate particles.

Administration of cycloheximide, by inhibiting chylomicron formation during the transport of glycerides from the terminal web to the endoplasm, induces immediate glyceride accumulation. Particulate fat increases up to Io $\mu$ diameter, causing the swelling and breaking of the reticular wall. The whole absorptive cell is gradually suffused with glycerides and comes to resemble an adipose cell. However, the Golgi complex and intercellular space do not contain any lipids. The cell elements are driven outside the cell or constricted between the glycerides. At one stage, the formation of dense bodies and the occurence of many fine-structural alterations were noticed. There were no chylomicrons in the stroma of the villi.

\section{RÉFÉRENCES BIBLIOGRAPHIQUES}

BAXTER J.H., 1966. Origin and characteristics of endogenous lipid in thoracic duct lymph in rat. J. Lipid Res., 7 , 158 -166.

Bergström S., Borgström B., Cartsten A., 1954. On the mechanism of intestinal fat absorption in the cat. Acta physiol. scand., 32, 94-98.

Blomstrand R., Ahrens E. H. Jr, 1958. The absorption of fats studied in a patient with chyluria. J. biol. Chem., 233, $32 \mathrm{r}-326$.

Blomstrand R., DAHLBÄCK O., Linder F., I959. Asymetric incorporation of linoleic acid-I-C14 and stearic acid-I-C ${ }^{14}$ into human lymph lecithins during fat absorption. Proc. Soc. exp. Biol. N. Y., 100, 768-771.

BoRGSTRÖM B., 195I. On the mechanism of the intestinal fat absorption. II. Acla chem. scand., 5, 643-646.

BorgströM B., 1960. Metabolism of glycerides. In BLOCK K., Lipid Metabolism, J. Wiley and Sons, New York, I 28-164.

Bragdon J. H., i 958 . On the composition of chyle chylomicrons. J. Lab. clin. Med., 52, 564-570.

BRAGDON J.H., KARMEN A., I 960 . The fatty acid composition of chylomicrons of chyle and serum following the ingestion of different oils. J. Lipid. Res., 1, I67-1 70 .

Burr W. W. Jr., McPherson J. C., Tidwell H. C., I960. Secretions of labeled blood lipids into the intestine. J. Nutr., 70, I7 $7^{-1} 75$.

CASLEY-Smith J. R., 1962. The identification of chylimicra and lipoproteins in tissue sections and their passage into jejunal lacteals. J. Cell. Biol., 15, $259^{-2} 77$.

CLÉment G. E., MEAD J. F., 1959. Study of lymph lipids following administration of oleic-I-C'14 acid with or without cholesterol. Proc. Soc. exp. Biol. N. Y., 100, 285-288.

Constantin M. J., Savary P., 1965. Sur l'incorporation des chaînes stéariques dans les chylomicrons lymphatiques du rat. Biochim. biophys. Acta, 106, 248-260.

COUREL E., ClEment J., 1964. Influence de l'ingestion de crème sur le nombre et la taille des chylomicrons lymphatiques. C. R. Soc. Biol., 158, 7 7 5-718.

DoBbins W. O., 1966. An ultrastructural study of the intestinal mucosa in congenital $\beta$-lipoprotein deficiency with particular emphasis upon the intestinal absorptive cell. Gastroenterology, 50, 195-210.

Dole V. P., Hamlin J. T., I962. Particulate fat in lymph and blood. Physiol. Rev., 42, 674-70I. 
Forstner G. G., Riley E. M., Daniels S. J., Isselbacher K. J., i 965. Demonstration of glyceride synthesis by brush borders of intestinal cells. Biochem. biophys. Res. Comm., 21, 83-98.

Franks J. J., Riley E. M., Isselbacher K. J., I966. Synthesis of fatty acids by Rat intestine in vitro. Prac. Soc. exp. Biol. Med., 121, 322-327.

Frezal J., Rey J., Polonowski J., Levy G., Lamy M., I96r. L'absence congénitale de $\beta$-lipoprotéines. Étude de l'absorption des graisses après exsanguino-transfusion. Mesure de la demi-vie des $\beta$-lipoprotéines injectées. Rev. franc. Étud. clin. biol., 6, 677-683.

GAGE S. H., I920. The free granules (chylomicrons) of the blood as shown by the dark field microscope. The Cornell Veterinarian, 10, I $54^{-1} 55$.

GAGE S. H., Fish P. A., 1924. Fat digestion, absorption and assimilation in man and animals as determined by the dark field microscope, and a fat-soluble dyes. Am. J. Anat., 84, 1-85.

Guiliver G., I840, cité par GaGe H., Fish P. A., 1924.

Hatch F. T., Aso Y., Hagopian L. M., Rubenstein J. J., ig66. Biosynthesis of lipoprotein by rat intestinal mucosa. J. biol. Chem., 241, I655-665.

IsSelbacher K. J., I967. Biochemical aspects of lipid malabsorption. Fed. Proc., 26, 1420-1425.

ISSELBACHER K. J., Budz D. M., 1963. Synthesis of lipoproteins by rat intestinal mucosa. Nature, London, (4904), 364-365.

Issetbacher K. J., Scheib R., Blotkin G. R., Gaulfield., 1964. Congenital $\beta$-lipoprotein deficiency : an hereditary disorder involving a defect in the absorption and transport of lipids. Medicine (Baltimore), 43, $347-37$ I.

Karmen A., Whyte M., Goodman D. S., I963. Fatty acid esterification and chylomicron formation during fat absorption. I. Triglycerides and cholesterol esters. J. Lipid. Res., 4, 31 2-321.

KAYDEN H. J., MEDICK M., 1967. Alternative pathways of long chain fatty acid absorption in the absence of chylomicron formation.' f. clin. Invest., 46, 1077 .

LaURell C. B., I953. Composition of chylomicrons isolated from rat's lymph. Acta physiol. scand., 80, 289-294.

LAURell C. B., 1954. Preliminary date on the composition and certain properties of human chylomicrons. Scand. J. Clin. Lab. Invest., 6, 22-24.

LeVy R. I., FREDRICKSON D. S., LASTER L., i 966. The lipoproteins and lipid transport in abetalipoproteinemia. J. clin. Invest., 45, 53 I-54I.

Lindgren F. T., Nichols A. V., rg6o. Structure and function of human serum lipoproteins. The Plasma proteins 11. Biosynthesis, metabolism, alterations in disease, F. W. Putnam ed., 1960, 23.

LUFT J., I96r. Improvements in epoxy resin embedding methods. J. biophys. biochem. Cytol., 9, 409-4I4.

Matsion F. H., Volpenhein R. A., 1964. The digestion and absorption of triglycerides. J. biol. Chem., 289, $2772-2777$.

MrLlonig G., I 961. The advantages of a phosphate buffer for $\mathrm{OsO}_{4}$ solutions in fixation. J.appl. Phys., 82, I637.

Merrill T. G., Sprinz N., Tousimis A. J., I967. Changes of intestinal absorption cells during maturation : an electron microscopic study of prenatal, postnatal and adult guinea pig ileum. J. Ultrastr. Res., 19, 304-326.

Nordstrom C., Dahlovist A., Josefson L., ig67. Quantitative determination of enzymes in different parts of the villi and crypts of rat small intestine. Comparison of alkaline phosphatase, disaccaridases and dipeptidases. J. Hislochem. Cylochem., 15, 713-721.

Pessoa V. C., KIM K. S., Ivy A. C., 1953. Fat absorption in absence of bile and pancreatic juice. Amer. J. Physiol., 172, 209-218.

Pinter G. G., Zilversmit D. B., 1962. A gradient centrifugation method for the determination of particle size distribution of chylomicrons and of fat droplets in artificial fat emulsion. Biochim. biophys. Acta, 59, II 6-I 27.

QUARFORDT S. H., GoODMAN D.S., I 966 . Heterogeneity in the rate of plasma clearance of chylomicrons of different size. Biochim. biophys. Acta., 116, $3^{82-3^{85}}$.

REY J., I961. L'absence congénitale de bêta-lipoprotéines. Thèse, Paris, I 8 pages.

REynolds E., 1963. The use of lead citrate at high $\mathrm{pH}$ as an electron opaque stain in electron microscopy. J. cell. Biol., 17, 208-2I 2.

Rodbell M., Frederickson D. S., ONo K., I959. Metabolism of chylomicron proteins in the dog. J. biol. Chem., 284, 567-571.

Rodbell M., Frederickson D. S., 1959. The nature of the proteins associated with dog and human chylomicrons. J. biol. Chem., 234, 562-566.

Roheim P. S., Gidez L. I., Eder H. A., 1966. Extrahepatic synthesis of lipoproteins of plasma and chyle role of the intestine. J. clin. Invest., 45, 297-300.

Saresin S., Isselbacher K. J., 1965. Protein synthesis inhibition : mechanism for the production of unpaired fat absorption. Science, 147, I $149-1151$.

Savary P., Constantin M. J., Desnuelle P., ig6r. Sur la structure des triglycérides des chylomicrons. lymphatiques du rat. Biochim. biophys. Acta., 48, 562-571. 
Savary P., Constantin M. J., I 966. Sur la résoprtion intestinale des chaînes éruciques et leur incorporation dans les chylomicrons lymphatiques du rat. Biochim. biophys. Acta, 125, 1 18-128.

SCANU A., PAGE I. H., 1959. Separation and characterization of human serum chylomicrons. J. exp. Med., 109, 236-239.

Shrivastava B. K., Redgrave T. G., Simmonds W. J., I966. The source of endogenous lipid in the thoracic duct lymph of fasting rats. Quart. J. exp. Physiol., 52, 305-312.

SJÖSTRAND F. S., BoRGSTRÖM B., I 967 . The lipid components of the smooth surfaced membrane bounded, vesicles of the columnar cells of the rat intestinal epithelium during fat absorption. J. Ullrastruct. Res. 20, $140-160$.

Thomas J. R., ScotT W. A., 1962. Electron microscopy study of chyle from rats fed butter or corn oil. Exp. mol. Path., 1, 65-83.

Trakatellis A. C., Montjar M., AXelrod A. E., 1965. Effect of cycloheximide on polysomes and protein synthesis in the mouse liver. Biochemistry, 4, 2065.

Verzar F., Mcdovgall E., 1936. Absorption from the intesine. Longmans, London, New York, 264 pages.

Vodovar N., Flanzy J., 1966. Mécanisme de la pénétration et voie d'acheminement des graisses dans les cellules absorbantes de l'intestin grêle du Porc. Ann. Biol. anim. Bioch. Biophys., 7, 31 5-332.

Vodovar N., FLANZY J., I 967 . Voie de pénétration des lipides ingérés dans le stroma des villosités. $C . R$. Acad. Sci., Paris 264, 850-853.

Vodovar N., Flanzy J,, Franç̧ors A.-C., 1967. a) Aspect morphologique de la répartition chyloportale des acides gras absorbês au niveau de l'intestin grêle. C. $R$. Acad. Sci., 265, 564-567.

Vodovar N., Flanzy J., FrançoIs A.-C., 1967. b) Répartition des acides gras ingérés entre vaisseaux sanguins et lactéales centrales dans le stroma des villosités. Ann. Biol. anim. Bioch. Biophys., 7, 423-435.

VODOVAR N., FuÉCHON J., I 966 . La cellule épithéliale absorbante de l'intestin grêle du porc. Ultrastructure. Ann. Biol. anim. Bioch. Biophys., 6, х2-32.

Vodovar N., Massicard N., Flanzy J., ig68. Formation et rôle des chylomicrons au cours de l'absorption des acides gras à chaîne longue. C.R. Acad. Sci., Paris, 268, 814-817.

WAYS P. O. et al., 1967 . Studies on the absorption defect for triglyceride in abetalipoproteinemia. J. clin. Invest., 46, 35-46.

Weld C. B., I96i. The enterolipomicron, a secretion. Canad. J. Biochem. Physiol., 39, I5-29.

Yokoyama A., Zilversmiz D. B., 1965. Particle size and composition of dog lymph chylomicrons. $J$. Lipid Res., 6, 241-246.

ZilvERSMIT D. B., ig65. The composition and structure of lymph chylomicrons in dog, rat and man. $J$. clin. Invest., 44, $1610-1622$.

Zilversmit D. B., Sisco P. H., Yokoyama A., 1966. Size distribution of thoracic duct lymph chylomicrons from rats fed cream and corn oil. Biochim. biophys. Acta, 125, I $29^{-1} 35$.

Zilversmit D. B., 1967. Formation and transport of chylomicrons. Fed. Proc., 26, 1599-1605.

\section{PLANCHE I}

Lieu de formation des chylomicrons lors de l'absorption normale des acides gras à chaîne longue (fig. I à 5)

FIG. I. - Coupe perpendiculaire au grand axe de la cellule absorbante dans la région supranucléaire L'incidence des coupes sur les particules lipidiques (L) est plus ou moins perpendiculaire à l'axe du RER. Cela a pour conséquence l'épaisseur variable de la bordure opaque ( $x 45000)$ 


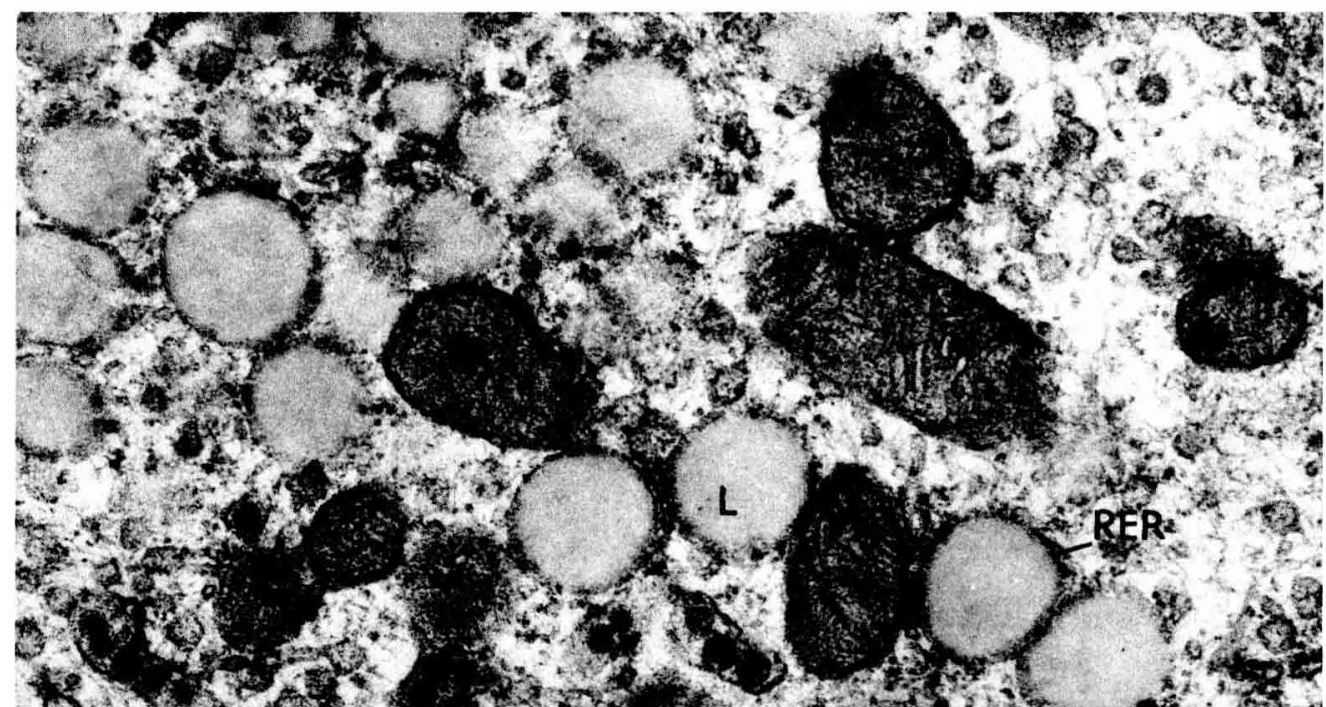

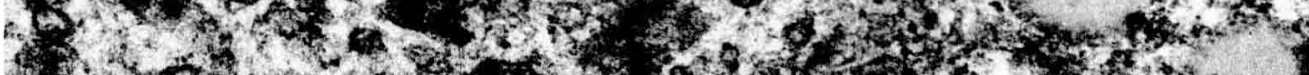

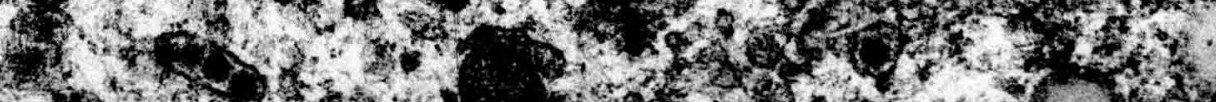

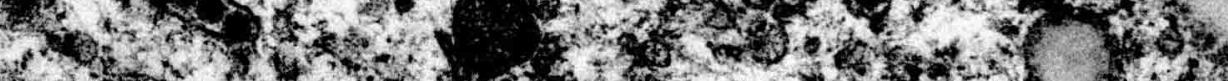

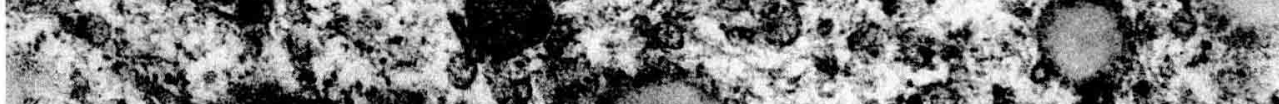

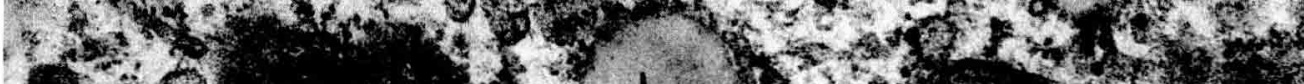

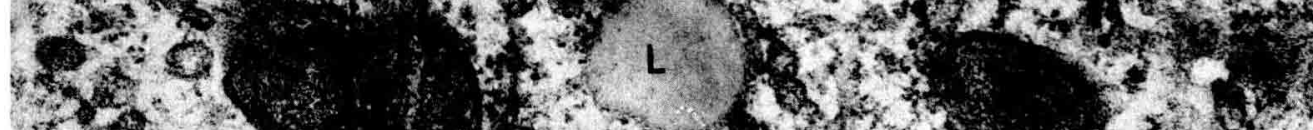

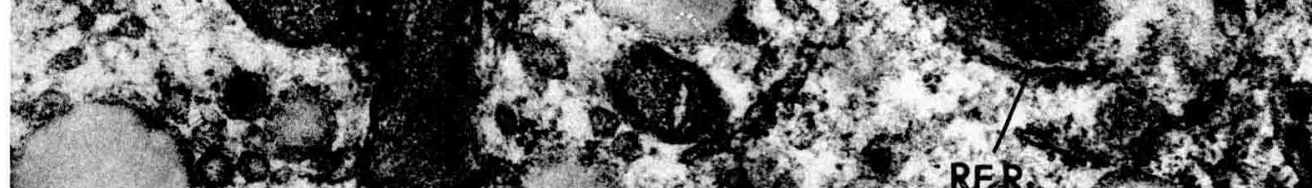

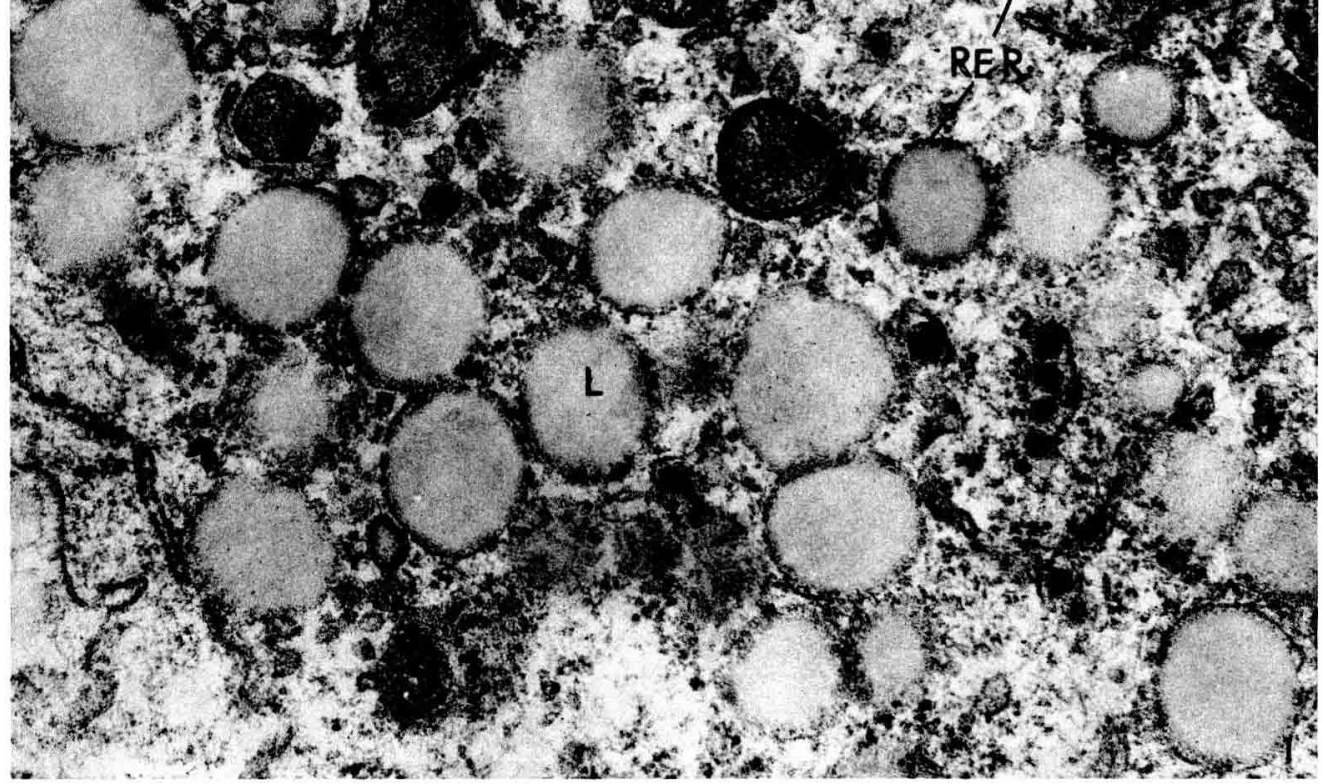

N. VODOVAR, J. FLANZY, A.-C. FRANÇOIS. 


\section{PLANCHE II}

Fig. 2. - Coupe de la régión golgienne. Les particules lipidiques nombreuses dans les vésicules golgiennes et entourées d'une enveloppe (EC), qui préserve leur intégrité, sont les cholymicrons. ( $x$ 30.000).

Fig. 3. - Coupe dans les zones apicale et subapicale grossièrement parallèle au grand axe de la cellule absorbante de l'intestin et aux axes du RER. On distingue les particules lipidiques (L) à l'intérieur du RER. Les particules dont la partie de surface n'est pas en contact avec la membrane réticulaire ont une enveloppe opaque (EC) à leur surface. $(\times 30.000)$. 


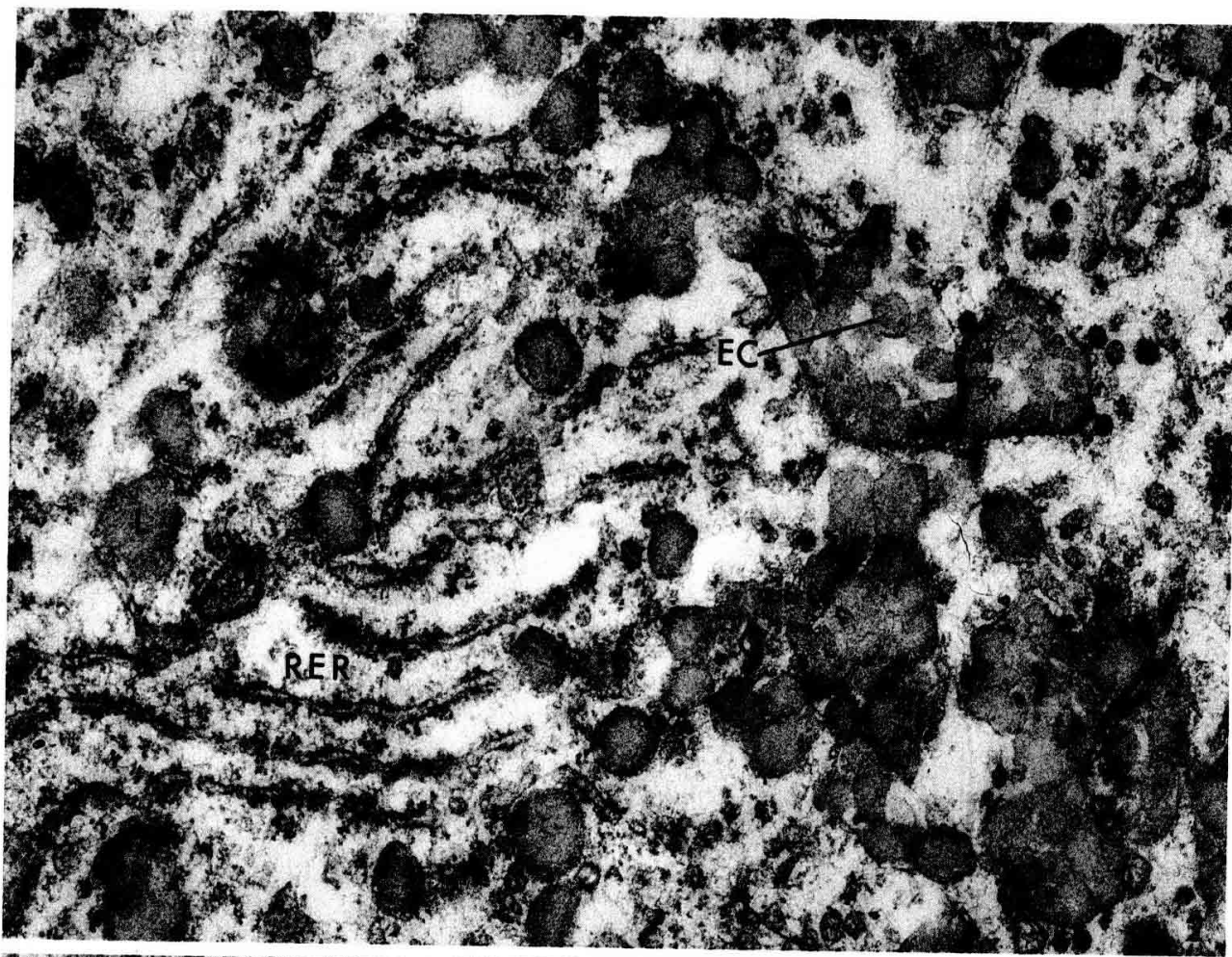

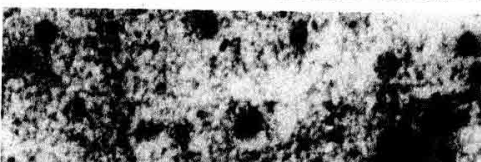

M.

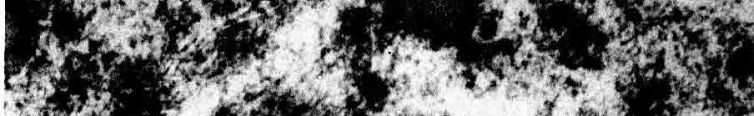

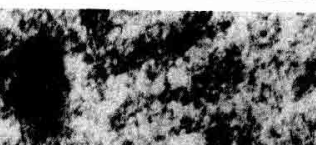

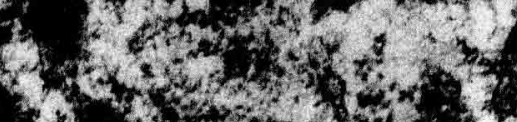

$x^{x} x^{2}$

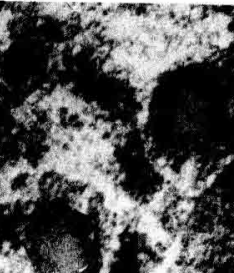

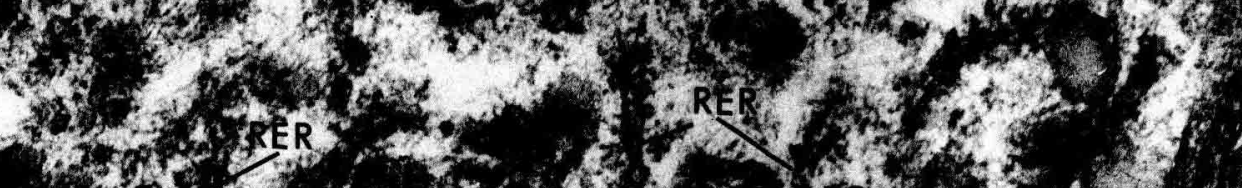

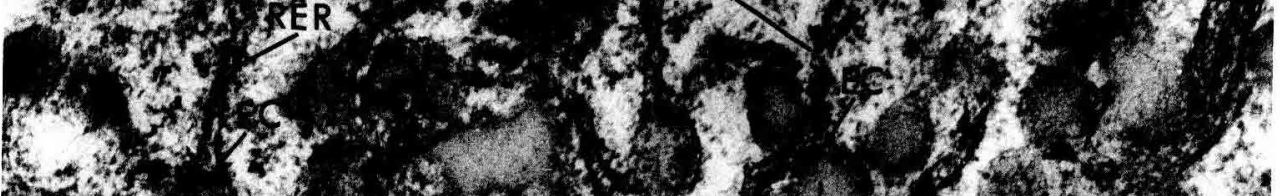

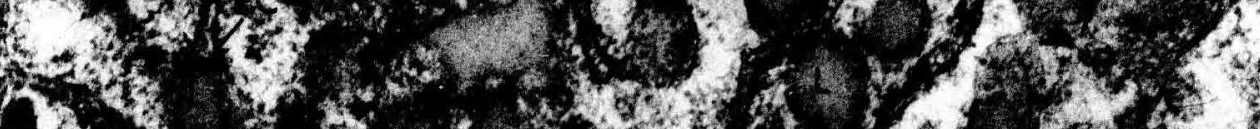

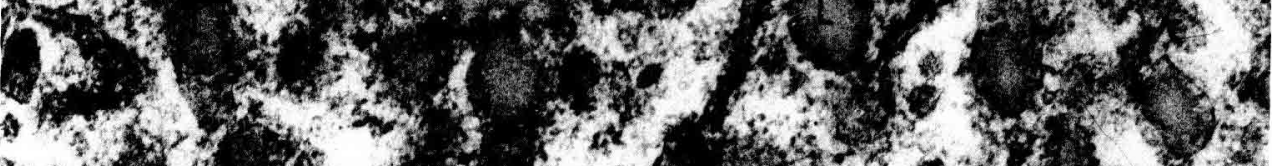
6.

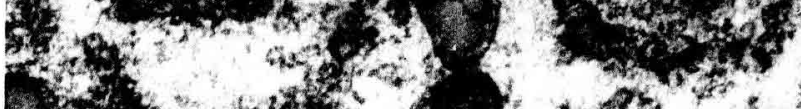

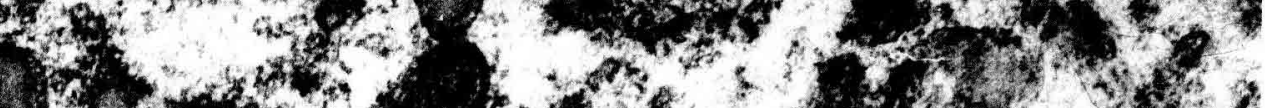

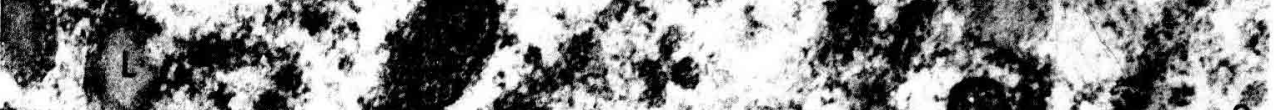

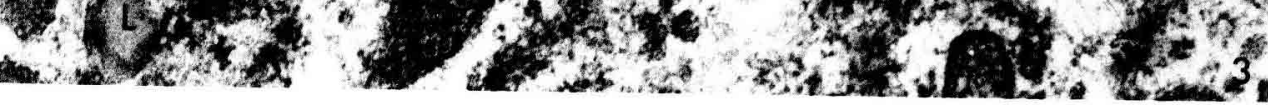

N. VODOVAR, J. FLANZY, A.-C. FRANÇOIS. 


\section{PLANCHE III}

Fic. 4. - Les gouttelettes lipidiques (L) dans la zone subapicale ont leur surface entierement ou partiellement en contact avec le RER. Dans le dernier cas, on observe sur la surface libre des gouttelettes une enveloppe opaque (EC), qui caractérise les chylomicrons. $(\times 25.000)$.

Fia. 5. - Mêmes éléments que sur la fig. 4 avec présence dans les espaces intercellulaires de chylomicrons (CH) entourés de l'enveloppe opaque. $(\times 25.000)$. 




N. VODOVAR, J. FLANZY, A.-C. FRANÇOIS. 
Aspect morphologique des difiérentes régions de la cellule absorbante de l'intestin au cours de l'absorption des acides gras à chaine longue après administration de cycloheximide. (fig. 6 ă 9).

\section{PLANCHE IV}

Fig. 6. - Zones apicale et subapicale, lipides (L) encore a l'intérieur du RER mais celui-ci est trés distendu. Corps denses (Ly) (lysosomes ?); les ribosomes (R) semblent souvent détachés du réticulum et parfois agglomérés. $(\times 30.000)$. 


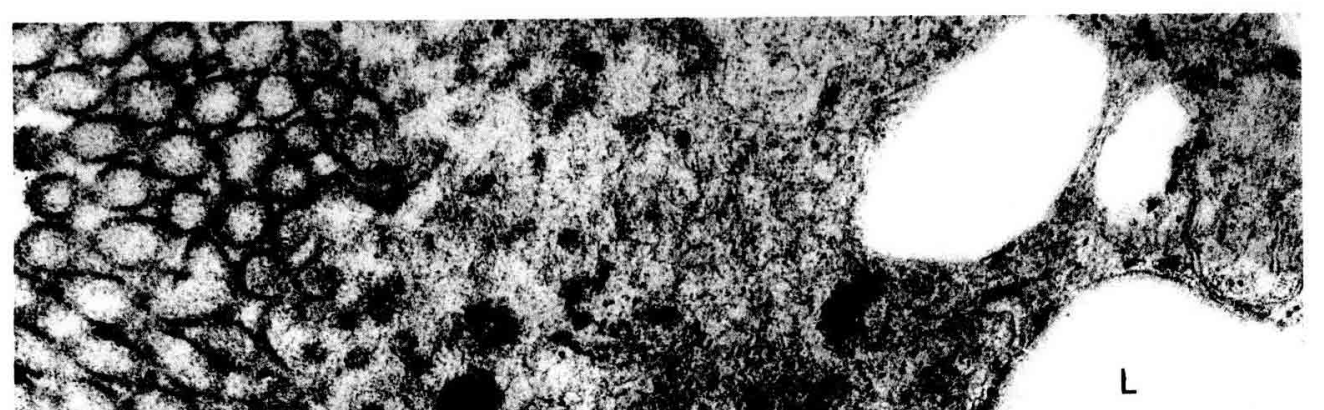

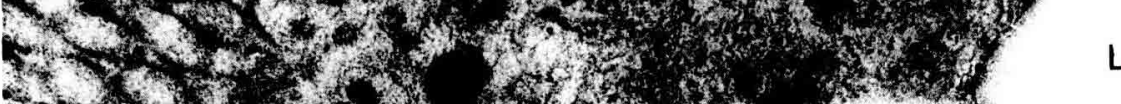

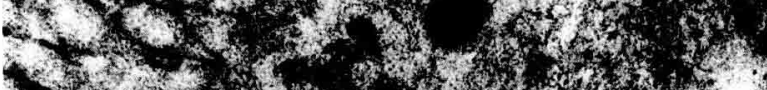

6.

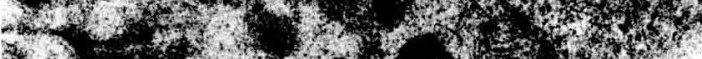

to m. H.t.
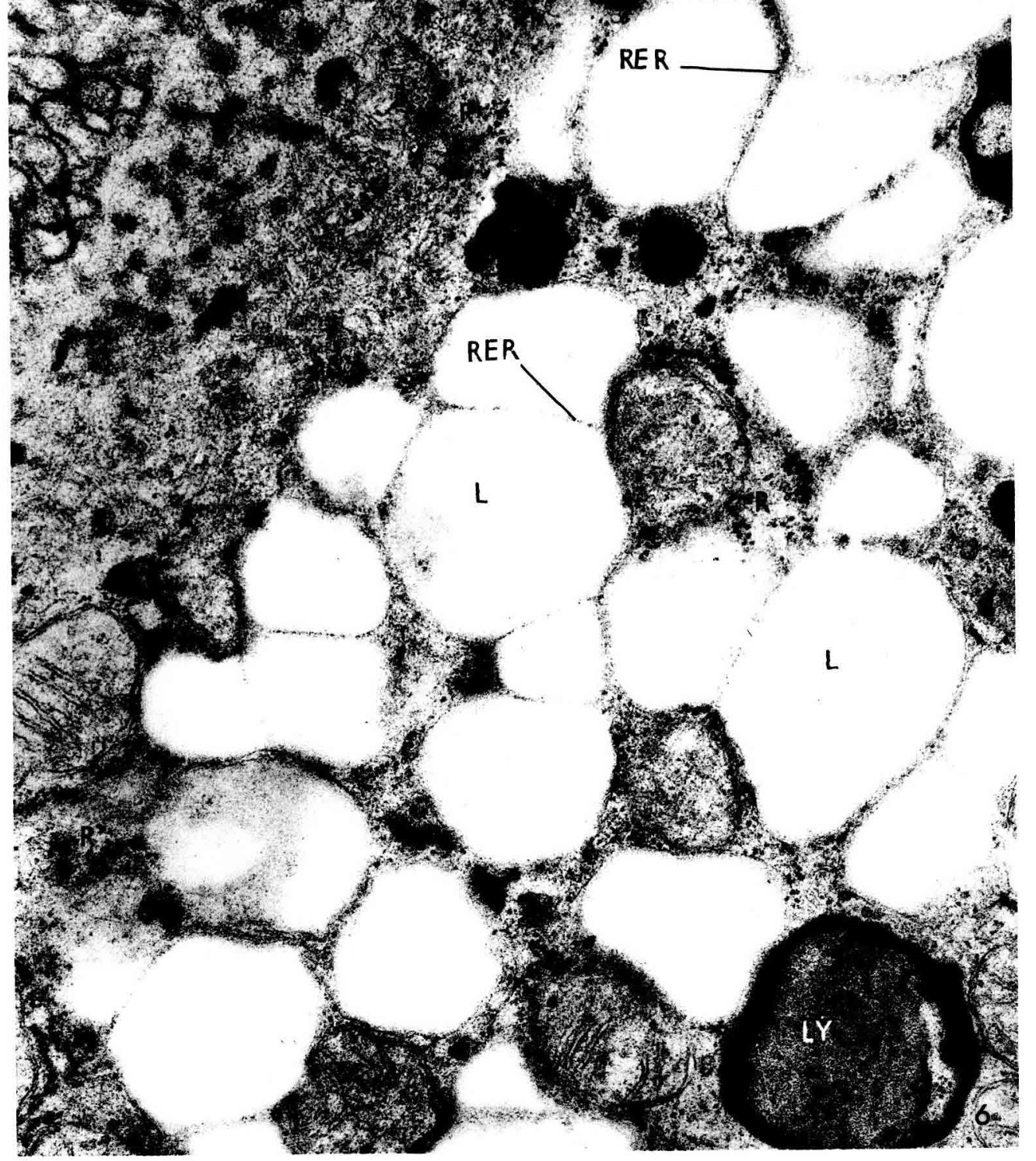

N. VODOVAR, J. FLANZY, A.-C. FRANÇOIS. 


\section{PLANCHE V}

Fıg. 7. - Appareil de Golgi (G) comprimé, les vésicules golgiennes sont pratiquement vides; mitochondries $(M)$ isolées et comprimées par les triglycérides. Espaces intercellulaires (El) sans lipides et peu importants. $(\times 20.000)$. 
Ann. Biol. anim. Bioch. Biophys., 1969, 9 (2).

Pl. V

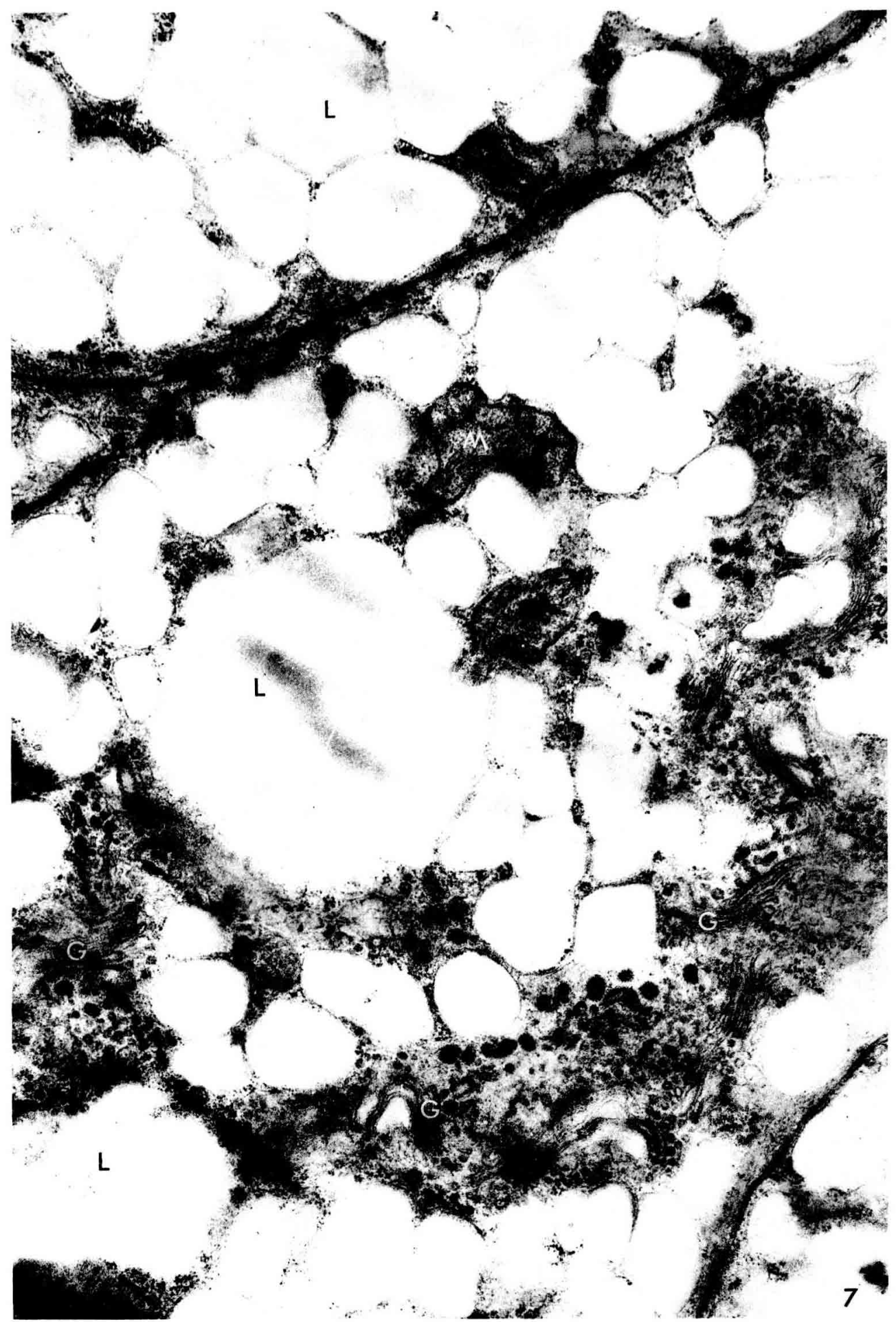

N. VODOVAR, J. FLANZY, A.-C. FRANÇOIS. 


\section{PLANCHE VI}

FIG. 8. - Région périnucléaire remplie de lipides (L), noyau (N) multilobé, entouré entièrement et serré par les lipides dont les grosses particules sont encore, en partie, séparées par le RER. Pas de lipides dans les espaces intercellulaires $(\mathrm{EI})$. $(\times 30.000)$. 


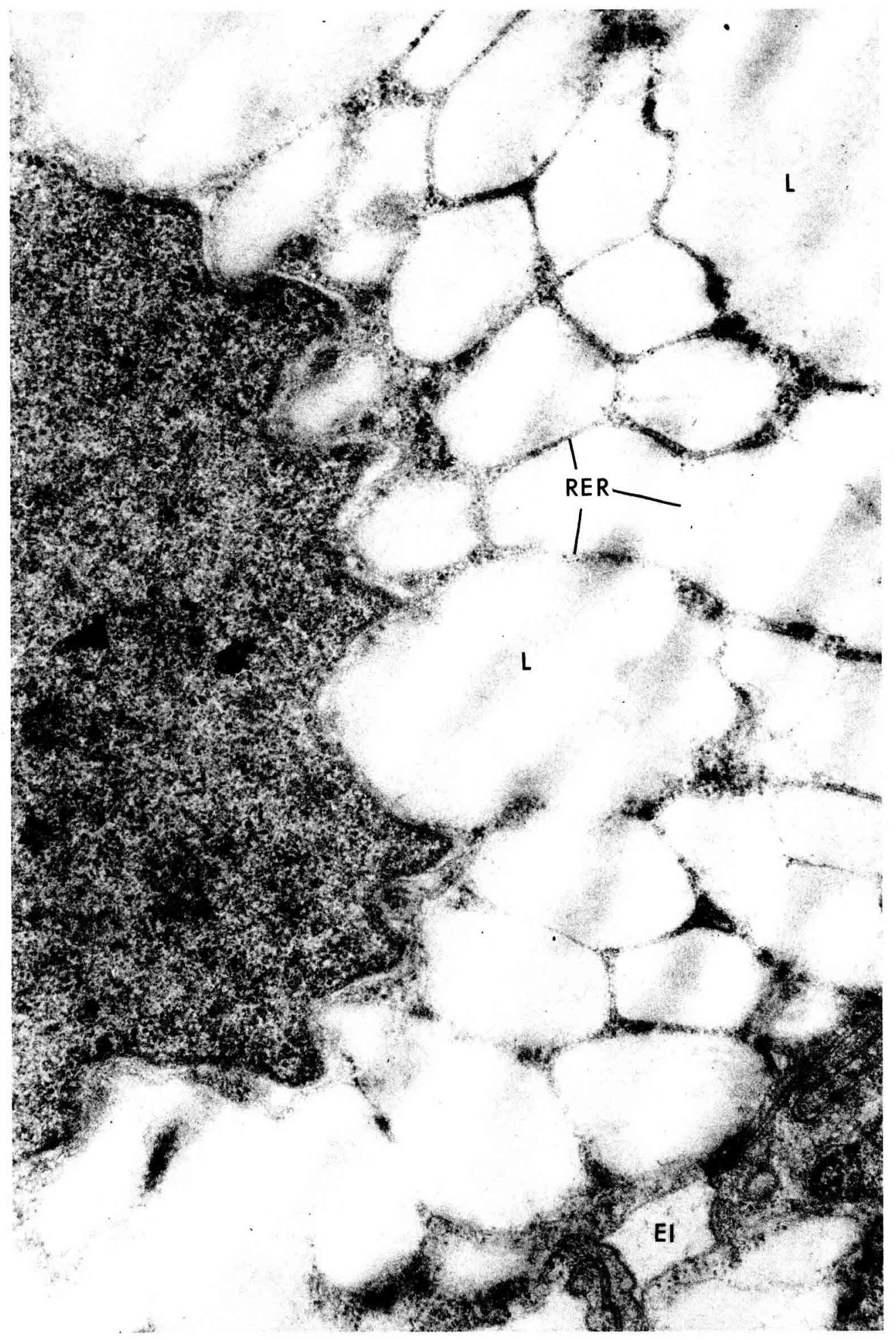

N. VODOVAR, J. FLANZY, A.-C. FRANÇOIS. 


\section{PLANCHE VII}

Fıc. 9. - Aspect partiel de trois cellules absorbantes contigués, remplies de lipides (L). Les éléments cytoplasmiques sont, soit comprimés entre les lipides, soit repoussés vers la périphérie; les parois réticulaires (RER) sont déja rompues ou distendues et prêtes a se rompre. Espaces intercellulaires (EI) réduits et sans lipides. Apparition de lysosomes (Ly). ( $\times$ 27.000). 


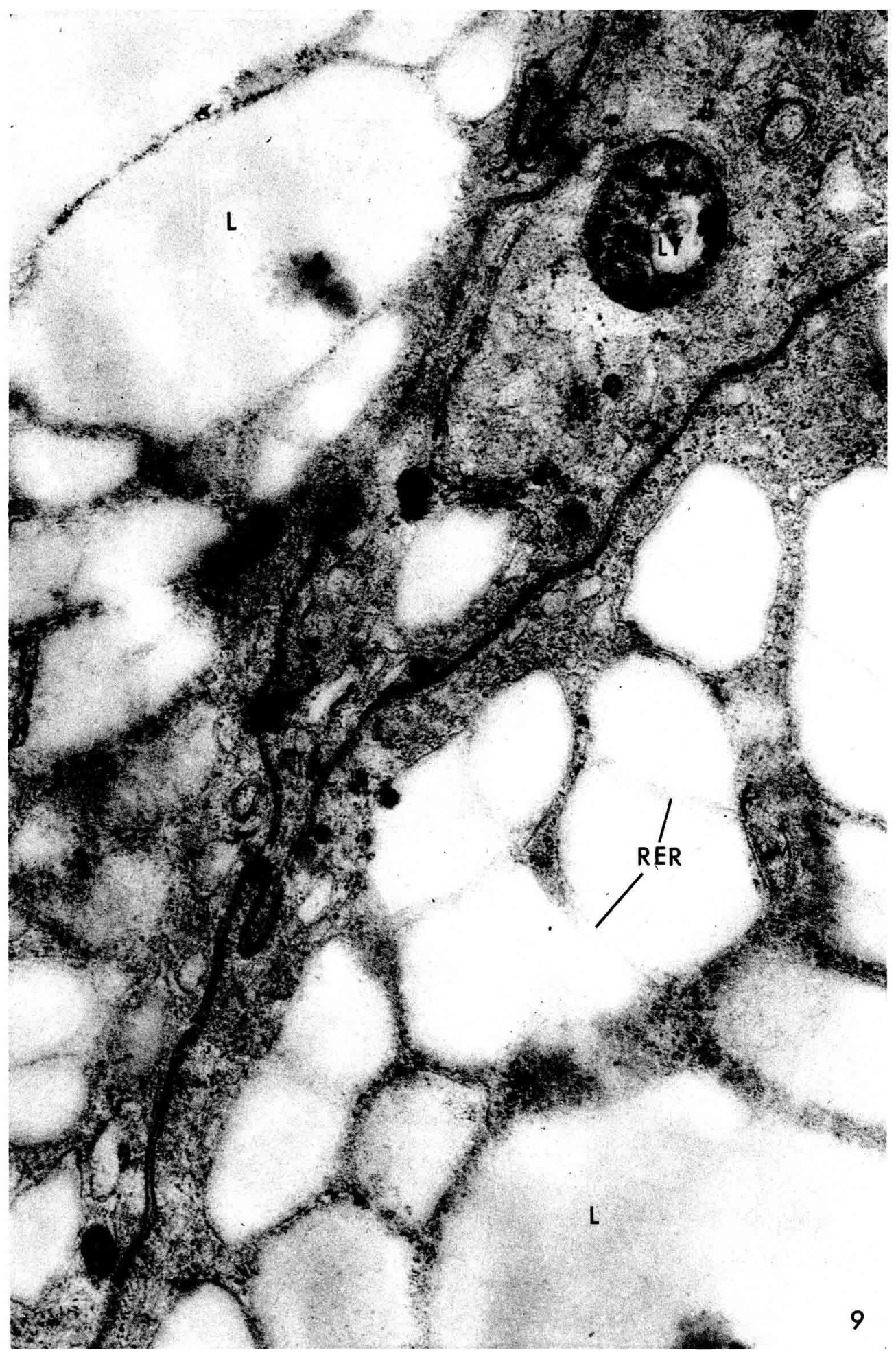

N. VODOVAR, J. FLANZY, A.-C. FRANÇOIS. 


\section{PLANCHE VIII}

Fig. 10 et 11. - Accumulation provoquée des chylomicrons (CH) dans la cellule absorbante au cours de l'absorption des acides gras à chaîne longue. L'aspect est très différent de celui des fig. 6 a 9 .

Fig. $10=33.000$.

Fig. $11=40.000$. 

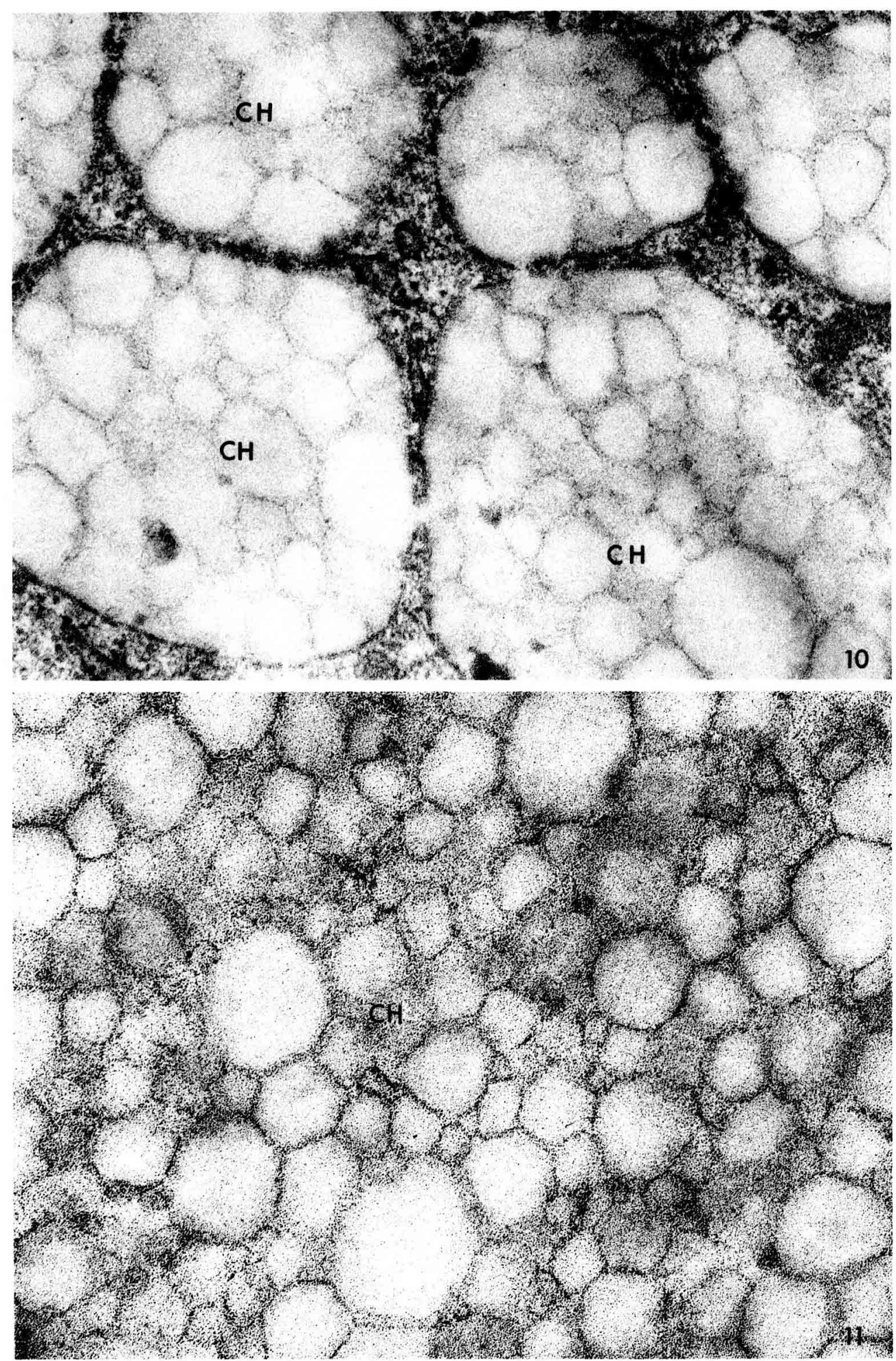

N. VODOVAR, J. FLANZY, A.-C. FRANÇOIS. 